\title{
Effect of Frame Structure on Interpretation of Map Symbols
}

\author{
Li, Zhilin ${ }^{\mathrm{a}, *}$
}

a The Hong Kong Polytechnic University

* e-mail: 1szlli@polyu.edu.hk

Keywords: Relational structure, topological structure, map symbol, symbol interpretation, frame of symbol

Various studies (e.g. Forrest and Castner 1985, Blok 1987, Clarke 1989, Leung and Li 2002) have shown that some map symbols are very effective (easy to understand the meaning) while some other symbols are not very effective (difficult to understand the meaning). Such results are in accordance our experiences in map use. A question arising is "What matters?" The simple answer is "complexity".

Map complexity is an important issue in cartography (Monmonier 1974, Dietzel 1983, Fairbairn 2006). Two types of complexity have been identified (Brophy 1980, MacEachren 1982), i.e., visual complexity (VC) and intellectual complexity (IC). Visual complexity, also called graphical complexity, is a direct consequence of the spatial recognition of the graphic content of the symbol. It is closely related to the visual impact of a symbol and to the perceptual processes of viewing it. Intellectual complexity, also called intrinsic complexity, is a result of the meanings or signification contained in or ascribed to the symbolism. It is related to the cognitive processes of understanding the symbol. As a result, based on the complexity, symbols can be classified in four types, i.e. low visual and intellectual complexity ( $\mathrm{VC}=$ low, IC=low), low visual but high intellectual complexity (VC=low, IC=high), high visual but low intellectual complexity $(\mathrm{VC}=$ high, $\mathrm{IC}=$ low $)$, and high visual and high intellectual complexity ( $\mathrm{VC}=$ high, $\mathrm{IC}=$ high). Some examples of symbols (downloaded from Internet) belonging to these categories are shown in Fig.1.

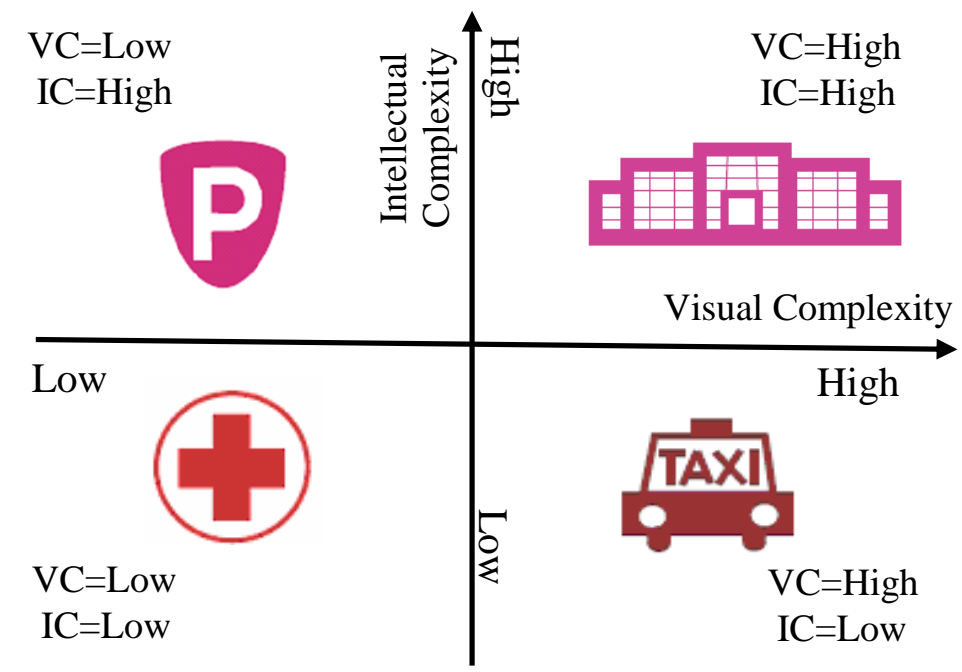

Fig. 1 Four types of map symbols based on complexity

In this figure, the red cross symbol looks simple and easy to understand. Almost 100\% of people interpret it correctly. The "P" symbol, representing "police station", looks simple but has been misinterpreted "post office", "car parking" and "parks" with $80 \%$ of people. The "taxi" symbol, although complex graphically, is easy to understand and is interpreted correctly by $100 \%$ of people. The "stadium" symbol is complex both visually and intellectually. It is surprising to notice that the percentage of correct interpretation is $0 \%$. The answers are "unknown", "industrial estate", "resort", or "shopping mall".

In our study, a symbol is decomposed into graphic components and the relationship between these components are described by spatial relations, e.g. topological relations, distance relations, order relations and directional relations. For example, the red cross symbol in Fig. 1 can be described by a topological structure, i.e. a circle as exterior, the red colour as interior and the cross boundary as symbol. It has been hypothesized that the relational structure will have effect on the interpretation of map symbols.

This paper discusses the effect of frame (i.e. exterior) on the interpretation of map symbols. A large number of map symbols have been tested and some interesting results have been obtained. For example, the three symbols as shown in Fig.2 are all "car park" symbols (downloaded from Internet), but they has been interpreted as different objects (features) due to the different shapes for the frame (exterior). For the left one, the rate of correct interpretation is 55\%; 40\% people answer "unknown" and 5\% people say "park". For the middle one, the rate of correct interpretation is $45 \%$; 20\% people 
answer "unknown" and 35\% people say "police station". For the right one, the rate of correct interpretation is 50\%; 20\% people answer "Park'N" supermarket and 15\% people say "police station". It means that frame with different shape may cause intellectual complexity increased.
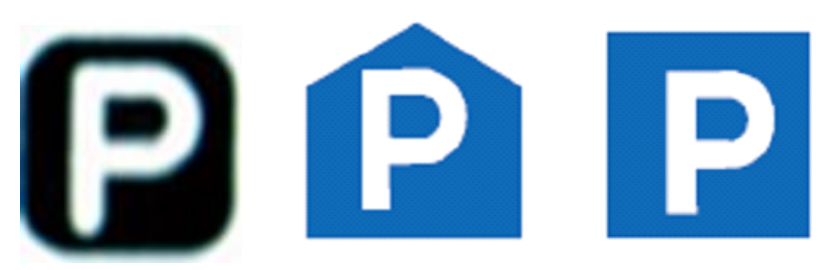

Fig.2 Three car park symbols with different exterior and thus with different intellectual complexity

Figure 3 shows another example (downloaded from Internet). These two are supermarket symbols. The left one is framed and the right is without any exterior. The rates of correct interpretation are $35 \%$ and $100 \%$ respectively. The left one is interpreted as shopping mall or "unknown". It means that the frame has significant effect on the intellectual complexity of map symbols.
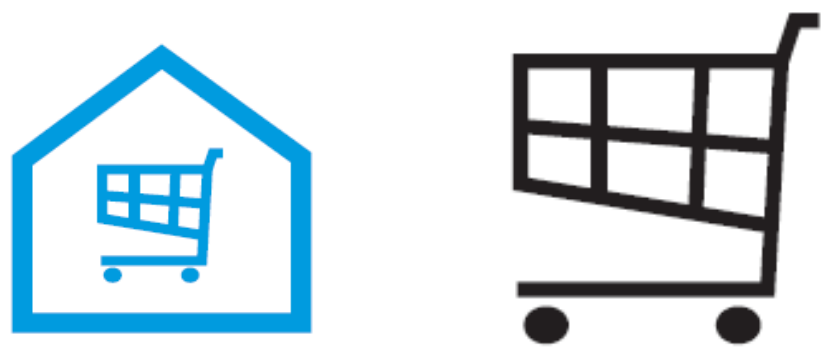

Fig.3 Two supermarket symbols with and without exterior, and thus with different intellectual complexity

In summary, extensive experimental tests have been to evaluate the intellectual complexity of map symbols and some interesting results have been obtained. It has been find that frame (or exterior) of a map symbol has significant impact on the intellectual complexity of map symbols and thus on the correct interpretation of map symbols. This may lead to a spatial-relation-based map symbol theory.

This research is supported by a grant from the Research Council of HKSAR (15223315).

Blok, C.A. (1987). Testing symbols on a Dutch tourist map, scale 1: 50.000. ITC journal, 1987(1): 67-72.

Brophy, D.M. (1980). Some reflections on the complexity of maps. Technical papers of ACSM 40th Annual Meeting, American Congress on Surveying and Mapping. 343-352.

Clarke, L.M. (1989). An experimental investigation of the communicative efficiency of point symbols on tourist maps. The Cartographic Journal, 26(2), 105-110.

Forrest, D. and Castner, H.W. (1985). The design and perception of point symbols for tourist maps. The cartographic journal, 22(1), 11-19.

MacEachren, A.M. (1982). Map complexity: comparison and measurement. The American Cartographer, 9(1), 31-46. doi:10.1559/152304082783948286

Monmonier, M.S. (1974). Measures of pattern complexity for choroplethic maps. The American Cartographer, 1(2), 159169. doi:10.1559/152304074784107728

Fairbairn, D. (2006). Measuring map complexity. The Cartographic Journal, 43(3), 224-238. doi:10.1179/ 000870406X169883

Dietzel, P.P. (1983). Measuring complexity on topographical maps. Proceedings of ACSM-ASP Fall Convention (pp. 45-49). Salt Lake City. Donderi, D. C. (2006). An information theory analysis of visual complexity and dissimilarity. Perception, 35(6), 823-835. doi:10.1068/p5249

Leung, L.F. and Li, Z.L. (2002). Experimental evaluation of the effectiveness of graphic symbols on tourist maps. Cartography, 31(1), 11-20. 